\title{
Methylammonium lead iodide: the crown prince of solar cell materials
}

\author{
Amlan J. Pal \\ In this article, important properties of methylammonium lead iodide responsible for its success as a \\ solar-cell material are discussed. We deliberate upon the rationale behind structural stability of \\ perovskite, its suitable optical properties, low exciton binding energy, solution processability, high \\ mobility, defect tolerance and low recombination loss, which have made the material truly special \\ and unique.
}

Keywords: Methylammonium lead iodide, perovskites, solar cell materials, structural stability.

IT is now a well-known fact that hybrid halide perovskites have exceeded as excellent solar cell materials. Methylammonium lead iodide $\left(\mathrm{CH}_{3} \mathrm{NH}_{3} \mathrm{PbI}_{3} ; \mathrm{MAPbI}_{3}\right)$ is indeed the crown prince in the materials-dynasty of solar cells, or may be in the materials world in general, since perovskite is being used in other electronic and optoelectronic applications as well ${ }^{1}$. In this article, we will deliberate upon the special properties of $\mathrm{MAPbI}_{3}$ that make it an ideal solar cell material.

\section{Structural stability}

$\mathrm{CH}_{3} \mathrm{NH}_{3} \mathrm{PbI}_{3}$ is a perovskite with an $A B X_{3}$ structure, where $A$ is a monovalent cation, $B$ is a bivalent metal and $X$ is a halogen. Each $B$-site cation is sixfold coordinated by the anions to form $B X_{6}$ octahedra. The methylammonium cation $\left(\mathrm{CH}_{3} \mathrm{NH}_{3}^{+}\right)$, which possesses a strong electric dipole, is surrounded by $\mathrm{PbI}_{6}$ octahedra. The octahedra are arranged in a 3D corner-sharing network, and each cavity of this network is occupied by one $A$-site cation ${ }^{2}$. In the cage of eight $\mathrm{PbI}_{6}$ octahedra, the size of the methylammonium cation is such that it can rotate inside $\mathrm{it}^{3}$. In

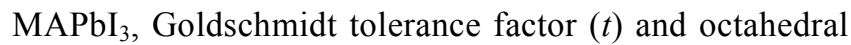
factor $(\mu)$, which are derived from effective ionic radii of the elements and are an indicator of the degree of stability and distortion in the crystal structure, are within the boundaries of stability range. This results in a stable cubic structure at high temperature, a tetragonal phase at intermediate temperature, and an orthorhombic phase at low temperature ${ }^{4}$. The tolerance and octahedral factors for $\mathrm{MAPbI}_{3}$ are well within the boundaries leaving further room for doping or alloying at each of the three sites (materials engineering), yielding a range of structurally

Amlan J. Pal is in the School of Physical Sciences, Indian Association for the Cultivation of Science, Jadavpur, Kolkata 700 032, India. e-mail:sspajp@iacs.res.in stable materials through multiple-cation, metal-substitution and halide-engineering approaches ${ }^{5}$.

\section{Optical bandgap}

The first aspect to consider in a stable solar cell material is its optical bandgap in connection to the ShockleyQueisser relation on power conversion efficiency (PCE) ${ }^{6}$. The relation as such provides an estimate of the maximum theoretical efficiency achievable from a singlejunction solar cell as a function of bandgap of the absorber; in such a plot, the maximum occurs at a bandgap of $1.34 \mathrm{eV}$ with a predicted maximum efficiency of $33.7 \%$. $\mathrm{MAPbI}_{3}$ has a bandgap of $1.55 \mathrm{eV}$, which is close to the optimum value ${ }^{5}$. More interestingly, the bandgap of $\mathrm{MAPbI}_{3}$ can be tuned through a substitution at each of the three sites through simple materials engineering. At present, with a bandgap of $1.55 \mathrm{eV}$, its intrinsic efficiency limit according to the Shockley-Queisser relation is $\sim 30.5 \%$.

The nature of bandgap in $\mathrm{MAPbI}_{3}$ is also ideal for many optoelectronic applications. First-principle calculations have shown that the minimum of conduction band (CB) and maximum of valence band (VB) both reside at the corner point ( $R$-point) in an $E$ - $\boldsymbol{k}$ space, leading to a direct bandgap for optical absorption ${ }^{7}$. Such a nature of band-edges in $\mathrm{MAPbI}_{3}$ can be mainly ascribed to the presence of local octahedral symmetry and a strong orbital coupling between lead and iodine ions. The organic moiety does not directly take part in the formation of band-edges; however, its steric effect influences the same to some extent ${ }^{8}$.

\section{High extinction coefficient}

Coupled with its near-optimum bandgap, $\mathrm{MAPbI}_{3}$ also displays a strong optical absorbance in the visible region 
of the electromagnetic spectrum owing to a high extinction coefficient. From a materialistic point of view, this feature has importance while designing a solar cell, as it reduces the thickness of the active layer required for absorbing adequate quanta of solar irradiation. In $\mathrm{MAPbI}_{3}$, high extinction coefficient is due to the nature of its bandgap, composition of CB and VB, and an associated transition between the band-edges ${ }^{8}$. First, due to direct bandgap in $\mathrm{MAPbI}_{3}$, the allowed transition occurs between the band-extremes. With respect to composition of the band-edges in $\mathrm{MAPbI}_{3}, \mathrm{CB}$ is primarily composed of unoccupied p-orbitals of lead $(\mathrm{Pb} 6 \mathrm{p})$, whereas $\mathrm{VB}$ is dominated by iodine p-orbitals (I 5p) along with some contribution from the s-orbitals of the metal $(\mathrm{Pb} 6 \mathrm{~s})^{9}$. Such a composition leads to a favourable $\mathrm{p}-\mathrm{p}$ transition between the band-edges and due to a less dispersive nature of p-orbitals, i.e. a high joint density of states, a strong optical absorption occurs in $\mathrm{MAPbI}_{3}$ (ref. 10)

\section{Solution processability and materials engineering}

Solution processability of materials for the formation of thin-film solar cells is a prerequisite for large area device fabrication. For perovskites, thin-film formation through a solution-cast route also provides opportunities to partially substitute an element with homovalent and heterovalent dopants, as long as $t$ and $\mu$ remain within the boundaries. In this respect, $\mathrm{MAPbI}_{3}$ is an ideal compound whose films can be casted through a range of solutionbased approaches, namely single-precursor or anti-solvent route, sequential deposition, Lewis acid-base adduct method, vapour-assisted solution process, etc. ${ }^{11}$. For example, $\mathrm{CH}_{3} \mathrm{NH}_{3} \mathrm{PbI}_{3}$ films can be casted in a glovebox from a stoichiometric amount of its precursors, namely $\mathrm{CH}_{3} \mathrm{NH}_{3} \mathrm{I}$ and $\mathrm{PbI}_{2}$ dissolved in a common solvent by thorough stirring. The films are then annealed at $100^{\circ} \mathrm{C}$ for about 20 min within the glovebox to complete the formation of $\mathrm{MAPbI}_{3}$.

Owing to this solution processability and unusual defect physics (vide infra), the $\mathrm{MAPbI}_{3}$ structure is flexible to support alloying at all the three ionic sites with suitable elemental or molecular analogues ${ }^{5}$. Hence, by tuning the composition in the solid solution, it is possible to identify a 'sweet spot' in achieving high solar cell efficiencies without compromising on the structural stability of the material. Methylammonium-site alloying plays a crucial role in dictating the dimension and stability of the perovskite structure with small influence on the optical bandgap. Inclusion of a large organic cation generally leads to a dimensional shrinkage, whereas inorganic cations help improve environmental stability. Lead-site alloying is primarily adopted to address the toxicity issue of $\mathrm{MAPbI}_{3}$; however, a suitable non-toxic alternative is yet to be found that can match the unique optoelectronic properties of the lead-based perovskite ${ }^{12}$. Since the halogen ions, depending upon their electronegativity, play a significant role in determining the bandgap of hybrid halide perovskites, mixed halogen compositions usually yield improved optical properties for the respective applications of the materials. In the $\mathrm{MAPbI}_{3}$ structure, it is possible to combine dual-site or triple-site alloying simultaneously in order to achieve multiple goals ${ }^{5}$.

\section{Low exciton binding energy}

Another unique feature of $\mathrm{MAPbI}_{3}$ is its low exciton binding energy. As such, this class of materials possesses a significantly larger dielectric constant $\left(\varepsilon_{r}>20\right)$ compared to conventional semiconductors due to lower crystal symmetry (centrosymmetry) and hence relative ease in polarizing the cell structure ${ }^{13}$. It also includes a rotational component associated with molecular dipole relaxation. The combination of a light effective carrier mass provided by the metal halide framework and a strong dielectric screening, including a molecule framework and an intermolecular interaction favour free-carrier generation over formation of excitons (bound electron-hole pairs) upon illumination. This leads to the origin of a significantly low exciton binding energy $(10-50 \mathrm{meV})$ in $\mathrm{MAPbI}_{3}$ (ref. 13). In other words, an electron and a hole are loosely bound due to a large dielectric constant and correspondingly a weak Coulomb interaction, guiding the carriers to reside in different molecules (Wannier exciton having a radius larger than the lattice spacing).

\section{High mobility and ambipolarity}

Organic semiconductors, which can arguably be considered as the predecessor of hybrid halide perovskites in the generation of solar-cell materials, did not succeed primarily due to a low carrier mobility in their thin films ${ }^{14}$. Again, as opposed to single crystals, thin films and solution processability are a prerequisite for large area device fabrication. The high carrier mobility in thin films of $\mathrm{MAPbI}_{3}$ can hence be considered as a key parameter for its success in optoelectronic applications, including solar cells. Theoretical studies have inferred enormously high electron and hole mobilities of 2800 and $9400 \mathrm{~cm}^{2} \mathrm{~V}^{-1} \mathrm{~s}^{-1}$ respectively; the experimental values recorded and reported so far are however below the estimates, leaving room for further improvement in $\mathrm{PCE}^{15}$. Such a high value of mobility arises presumably from a low effective mass of the carriers - about a tenth of the free electron mass ${ }^{10}$. Typically, band-edges derived from p-orbitals exhibit larger effective masses than those derived from s-orbitals, since the latter are more delocalized. Surprisingly, in the case of $\mathrm{MAPbI}_{3}$, although the $\mathrm{CB}$ and $\mathrm{VB}$ edges are predominantly formed through p-orbitals, a low effective mass of carriers is still obtained 
in perovskite. The reason for such uniqueness is the presence of a strong spin-orbit coupling (SOC) effect in $\mathrm{MAPbI}_{3}$ arising due to the high atomic number of lead $(Z=82)$. A strong hybridization (orbital overlap) in the band structures of $\mathrm{MAPbI}_{3}$, along with the octahedral framework result in such light effective mass of the carri$\mathrm{ers}^{16}$. The high mobility in turn leads to a long carrier diffusion length of around $100 \mathrm{~nm}$, ensuring hindrance-free carrier transport in a $\mathrm{MAPbI}_{3}$ layer.

The high mobility in $\mathrm{MAPbI}_{3}$ is further complemented by its ambipolar nature, making the class of materials truly unique. Ambipolarity, which is an interesting property for fundamental research and technological applications, is defined by a balanced electron and hole diffusion under the influence of an electric field. As mentioned earlier, estimation of electron and hole effective masses for $\mathrm{MAPbI}_{3}$ from the dispersion relation resulted in a closely matching value ${ }^{10}$. Due to the presence of methylammonium cation in $\mathrm{MAPbI}_{3}$, there exist $\mathrm{NH} \cdots \mathrm{I}$ long-rangebonding interactions which result in a favourable population of carriers specifically in the valence band maximum (VBM) and VBM coincides with the conduction band minimum $(\mathrm{CBM})$ at the $R$-point ${ }^{17}$. More evidence on the role of methylammonium cations on the ambipolar nature can be obtained by its removal from perovskite. A fully inorganic perovskite possesses a more uniform VBM, resulting in a marked electron transport character in perovskite $^{18}$.

\section{Defect tolerance}

Although $\mathrm{MAPbI}_{3}$ contains a large number of point defects due to its formation through common solution-based approaches, their presence does not affect the functional properties of the material. Depending on the stoichiometry of the precursors and activation energy of the defect, only one type of defect dominates in $\mathrm{MAPbI}_{3}$ : (1) metal vacancy when perovskite is formed in a $\mathrm{CH}_{3} \mathrm{NH}_{3} \mathrm{I}$-rich environment, or (2) iodine vacancy when the material is formed in $\mathrm{a} \mathrm{PbI}_{2}$-rich condition ${ }^{19}$. In either of the selfdoping cases, in-depth band-structure studies have shown that the material is highly defect-tolerant and hence the defects do not adversely affect the solar-cell performance ${ }^{20}$.

To understand the defect-tolerant nature of $\mathrm{MAPbI}_{3}$, it is imperative to know details of band formation in the material. In-depth analysis of band structures showed that $\mathrm{VB}$ and $\mathrm{CB}$ are formed with antibonding $\mathrm{Pb} 6 \mathrm{~s}-\mathrm{I} 5 \mathrm{p}$ and bonding $\mathrm{Pb} 6 \mathrm{p}-\mathrm{I} 5 \mathrm{p}$ molecular orbitals respectively. Due to the weak electronegative nature of iodide, its $5 \mathrm{p}$ orbitals are held loosely by the nucleus and therefore a strong interaction with the metal orbitals is expected. As a result, compared to other halogens, presence of iodine in $\mathrm{MAPbI}_{3}$ makes the bonding orbital (CB) more stabilized and the antibonding orbital (VB) more destabilized.
Therefore, under the influence of a strong $\mathrm{Pb} 6 \mathrm{~s}-\mathrm{I} 5 \mathrm{p}$ coupling, $\mathrm{VBM}$ in $\mathrm{MAPbI}_{3}$ is pushed to a high level so that the dominating acceptor levels (lead and methylammonium vacancies) become shallow ${ }^{21}$. On the other hand, the dominating donor-type vacancies such as methylammonium interstitials and iodine vacancies form shallow levels owing to the high ionicity of methylammonium and lead cations. Unlike deep-level defects that promote nonradiative recombination of charge carriers, shallow defects provide free carriers ${ }^{10}$. That is, the defects in $\mathrm{MAPbI}_{3}$ are somewhat 'inert' and do not have an adverse impact on the carrier diffusion length and carrier lifetime as well. This property hence provides an extra edge to perovskite compared to other known solar-cell materials such as kesterite $\left(\mathrm{Cu}_{2} \mathrm{ZnSnS}_{4}\right)$ or chalcopyrite $\left(\mathrm{CuIn}_{x} \mathrm{Ga}_{1-x} \mathrm{Se}_{2}\right)$, where bands form due to bonding orbitals; non-radiative recombination in those materials is therefore high due to defects formed within the bandgap ${ }^{22}$.

\section{Low recombination loss}

The most intriguing property of $\mathrm{MAPbI}_{3}$ is probably a low recombination rate of the photogenerated carriers. The recombination rate is five orders of magnitude lower than the predicted Langevin limit $^{23}$. It may be recalled that such a low rate occurs in $\mathrm{MAPbI}_{3}$ in spite of the films being formed through crude solution-processed routes, which in general result in a plethora of defects and impurities in other semiconductors leading to a high recombination loss in their thin films.

A low recombination rate of the photogenerated carriers in $\mathrm{MAPbI}_{3}$ enhancing the carrier lifetime and thereby carrier diffusion length is considered to occur due to Rashba band splitting ${ }^{24}$. In a nonmagnetic system like $\mathrm{MAPbI}_{3}$, the Rashba effect takes place due to a large spin-orbit coupling (SOC) parameter arising out of high$Z$ elements (lead and iodine) in the compound; moreover, the tilted $\mathrm{PbI}_{6}$ octahedra and rotating electric dipoles of methylammonium ions provide an effective magnetic field which lifts the Kramer's spin-degeneracy of the electrons ${ }^{25}$. Since splitting for CB and VB energies are non-identical and occur in the momentum $(\boldsymbol{k})$ space as well, the electrons and holes upon photoexcitation reside in a different $\boldsymbol{k}$-space. Their recombination, which now requires a phonon, hence becomes a slow process. Moreover, the two bands can be seen to split in such a manner that $\mathrm{CB}$ and VB, which are closer to the Fermi energy, have the same type of spin texture as proposed through a spin-allowed transition model ${ }^{26,27}$; such a splitting may apparently favour carrier recombination. Now, high dielectric constant of the material leading to low exciton binding energy results in Wannier-type excitons (electron and hole residing in different molecules). Therefore, instead of an intramolecular recombination, intermolecular transitions become the only possible route for carrier 
recombination; for an intermolecular recombination, different total angular momentum values of CBM in a molecule and VBM in a neighbouring molecule make the intermolecular transition a forbidden process, leading to a low recombination rate of the photogenerated carriers in $\mathrm{MAPbI}_{3}$.

\section{Conclusion}

Thus we have highlighted the important properties of $\mathrm{MAPbI}_{3}$ responsible for its success in solar cells. It is indeed rare to find a single semiconductor possessing several such favourable properties. The drawbacks in terms of stability can be surely circumvented through a suitable encapsulation. With a possibility of further improvement, researchers around the world now eagerly wait for coronation of $\mathrm{MAPbI}_{3}$ to the throne or in solar panels.

1. Extance, A., Perovskites on trial. Nature, 2019, 570(7762), 429432.

2. Fakharuddin, A., De Rossi, F., Watson, T. M., Schmidt-Mende, L. and Jose, R., Research update: behind the high efficiency of hybrid perovskite solar cells. APL Mater., 2016, 4(9), 091505.

3. Leguy, A. M. A. et al., The dynamics of methylammonium ions in hybrid organic-inorganic perovskite solar cells. Nature Commun., 2015, 6, 7124

4. Li, Z., Yang, M. J., Park, J. S., Wei, S. H., Berry, J. J. and Zhu, K., Stabilizing perovskite structures by tuning tolerance factor: formation of formamidinium and cesium lead iodide solid-state alloys. Chem. Mater., 2016, 28(1), 284-292.

5. Zhou, Y. Y., Zhou, Z. M., Chen, M., Zong, Y. X., Huang, J. S., Pang, S. P. and Padture, N. P., Doping and alloying for improved perovskite solar cells. J. Mater. Chem. A, 2016, 4(45), 1762317635.

6. Shockley, W. and Queisser, H. J., Detailed balance limit of efficiency of $p-n$ junction solar cells. J. Appl. Phys., 1961, 32(3), 510-519.

7. Leguy, A. M. A. et al., Experimental and theoretical optical properties of methylammonium lead halide perovskites. Nanoscale, 2016, 8(12), 6317-6327.

8. Huang, W. et al., Observation of unusual optical band structure of $\mathrm{CH}_{3} \mathrm{NH}_{3} \mathrm{PbI}_{3}$ perovskite single crystal. ACS Photonics, 2018, 5(4), 1583-1590.

9. Endres, J. et al., Valence and conduction band densities of states of metal halide perovskites: a combined experimental-theoretical study. J. Phys. Chem. Lett., 2016, 7(14), 2722-2729.

10. Xiao, Z., Song, Z. and Yan, Y., From lead halide perovskites to lead-free metal halide perovskites and perovskite derivatives. $A d v$. Mater., 2019, 31(47), 1803792.

11. Park, N. G., Methodologies for high efficiency perovskite solar cells. Nano Converg., 2016, 3, 15.

12. Chatterjee, S. and Pal, A. J., Influence of metal substitution on hybrid halide perovskites: towards lead-free perovskite solar cells. J. Mater. Chem. A, 2018, 6(9), 3793-3823.
13. Yang, Z. et al., Unraveling the exciton binding energy and the dielectric constant in single-crystal methylammonium lead triiodide perovskite. J. Phys. Chem. Lett., 2017, 8(8), 1851-1855.

14. Rolin, C., Kang, E., Lee, J. H., Borghs, G., Heremans, P. and Genoe, J., Charge carrier mobility in thin films of organic semiconductors by the gated van der Pauw method. Nature Commun., 2017, 8, 14975.

15. Mante, P. A., Stoumpos, C. C., Kanatzidis, M. G. and Yartsev, A., Electron-acoustic phonon coupling in single crystal $\mathrm{CH}_{3} \mathrm{NH}_{3} \mathrm{PbI}_{3}$ perovskites revealed by coherent acoustic phonons. Nature Commun., 2017, 8, 14398.

16. Even, J., Pedesseau, L., Jancu, J. M. and Katan, C., Importance of spin-orbit coupling in hybrid organic/inorganic perovskites for photovoltaic applications. J. Phys. Chem. Lett., 2013, 4(17), 29993005.

17. Giorgi, G. and Yamashita, K., Organic-inorganic halide perovskites: an ambipolar class of materials with enhanced photovoltaic performances. J. Mater. Chem. A, 2015, 3(17), 8981-8991.

18. Chen, Y. et al., Tuning the electronic structures of all-inorganic lead halide perovskite $\mathrm{CsPbI}_{3}$ via heterovalent doping: a firstprinciples investigation. Chem. Phys. Lett., 2019, 722, 90-95.

19. Wang, Q., Shao, Y. C., Xie, H. P., Lyu, L., Liu, X. L., Gao, Y. L. and Huang, J. S., Qualifying composition dependent $p$ and $n$ selfdoping in $\mathrm{CH}_{3} \mathrm{NH}_{3} \mathrm{PbI}_{3}$. Appl. Phys. Lett., 2014, 105(16), 163508.

20. Chen, B., Rudd, P. N., Yang, S., Yuan, Y. B. and Huang, J. S., Imperfections and their passivation in halide perovskite solar cells. Chem. Soc. Rev., 2019, 48(14), 3842-3867.

21. Yin, W. J., Shi, T. T. and Yan, Y. F., Unusual defect physics in $\mathrm{CH}_{3} \mathrm{NH}_{3} \mathrm{PbI}_{3}$ perovskite solar cell absorber. Appl. Phys. Lett., 2014, 104(6), 063903.

22. Kaur, K., Kumar, N. and Kumar, M., Strategic review of interface carrier recombination in earth abundant $\mathrm{Cu}-\mathrm{Zn}-\mathrm{Sn}-\mathrm{S}-\mathrm{Se}$ solar cells: current challenges and future prospects. J. Mater. Chem. A, 2017, 5(7), 3069-3090.

23. Wehrenfennig, C., Eperon, G. E., Johnston, M. B., Snaith, H. J. and Herz, L. M., High charge carrier mobilities and lifetimes in organolead trihalide perovskites. Adv. Mater., 2014, 26(10), 15841589.

24. Zheng, F., Tan, L. Z., Liu, S. and Rappe, A. M., Rashba spin-orbit coupling enhanced carrier lifetime in $\mathrm{CH}_{3} \mathrm{NH}_{3} \mathrm{Pbl}_{3}$. Nano Lett., 2015, 15(12), 7794-7800.

25. Kepenekian, M. and Even, J., Rashba and Dresselhaus couplings in halide perovskites: accomplishments and opportunities for spintronics and spin-orbitronics. J. Phys. Chem. Lett., 2017, 8(14), 3362-3370.

26. Zhang, X., Shen, J. X. and Van de Walle, C. G., Threedimensional spin texture in hybrid perovskites and its impact on optical transitions. J. Phys. Chem. Lett., 2018, 9(11), 2903-2908.

27. Maiti, A., Khatun, S. and Pal, A. J., Rashba band splitting in $\mathrm{CH}_{3} \mathrm{NH}_{3} \mathrm{PbI}_{3}$ : an insight from spin polarized scanning tunneling spectroscopy. Nano Lett., 2020, 20(1), 292-299.

ACKNOWLEDGEMENTS. I acknowledge my student Dr Soumyo Chatterjee, Research Associate, IACS for his help during preparation of the manuscript. In the financial front, JC Bose Fellowship (SB/S2/JCB001/2016) of SERB is acknowledged.

Received 24 April 2020; accepted 16 July 2020

doi: $10.18520 / \mathrm{cs} / \mathrm{v} 119 / \mathrm{i} 6 / 915-918$ 